\title{
A Novel Dihydropyridines C-Glycosylated Compound by Hantzsch Cyclo-Condensation
}

\author{
Soheila Sedaghat, Shabnam Madjdpour \\ Chemistry Department, Islamic Azad University, Tehran North Branch, Tehran, Iran \\ Email: s_sedaghat@iau-tnb.ac.ir
}

How to cite this paper: Sedaghat, S. and Madjdpour, S. (2018) A Novel Dihydropyridines C-Glycosylated Compound by Hantzsch Cyclo-Condensation. Open Journal of Synthesis Theory and Applications, 7, 17-29. https://doi.org/10.4236/ojsta.2018.72002

Received: February 4, 2018

Accepted: April 25, 2018

Published: April 28, 2018

Copyright $\odot 2018$ by authors and Scientific Research Publishing Inc. This work is licensed under the Creative Commons Attribution International License (CC BY 4.0).

http://creativecommons.org/licenses/by/4.0/

\section{cc) (i) Open Access}

\begin{abstract}
The Hantzsch synthesis has been used for the preparation of the 1,4-Dihydropyridines compounds with different pharmacophore groups. The use of a designed glycosylated compound in Hantzsch synthesis would lead to a novel Dihydropyridines C-Glycosylated compound. We used the 6-methoxy-2, 2-dimethyltetrahydrofuro $[3,4-\mathrm{d}][1,3]$ dioxole-4-carbaldehyde as a glycosylated-based aldehydes. The 4-(3,4-Dihydroxy-5-methoxy-tetrahydro-furan2-yl)-2,6-dimethyl-1,4-dihydro-pyridine-3,5-dicarboxylic acid 3-ethyl ester 5 -methyl ester was synthesized. The structure of compounds was determined by NMR spectroscopy, FTIR and mass spectroscopy methods. We synthesized this 1,4-Dihydropyridines compound by ionic liquid under ultrasound irradiation as a green chemistry synthesis.
\end{abstract}

\section{Keywords}

1,4-Dihydropyridines, c-Glycosylated, Multi Component Reaction, Hantzsch Reaction

\section{Introduction}

1,4-Dihydropyridines (1,4-DHPs) have attracted interest significantly because of their versatile pharmaceutical properties such as antibacterial and antitumor effects. The 1,4-DHPs have applied as a calcium channel blocker in cardiovascular disease. Some methods for the synthesis of 1,4-DHPs have been reported [1] [2] [3] [4] [5].
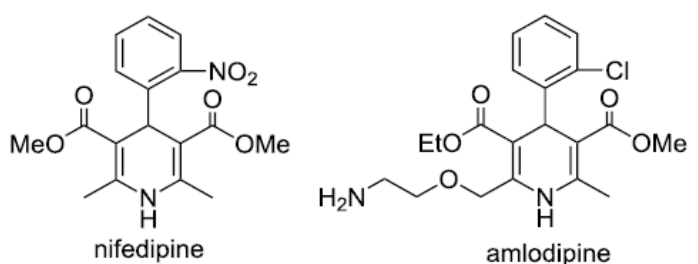
1,4-DHPs are synthesized by Hantzsch method which involves cyclo condensation of an aldehyde, $\beta$-diketone and ammonia in acetic acid which leads to derivatives of dihydropyridines. The synthesis of Dihydropyridines C-Glycosylated has importance because of their good pharmacokinetic and the role of compound in molecular recognition [6]. Dondoni has reported the synthesis and use of a designed glycosylated compound in some of the possible combinations in Hantzsch synthesis. He prepared them as a collection of C-nucleosides dihydropyridines compounds and some exhibiting significant biological activities have been reported [7] [8] [9] [10].

Ultrasound method improved some chemical reaction by a physical phenomenon named cavitation. Cavitation can contribute to the development of chemical reactions in the solution through a radical mechanism. There are the formation, growth, and collapse of bubbles in a medium. Compared to the traditional synthesis, this technique is more convenient by green chemistry concept [11] [12] [13] [14].

We synthesized substituted Dihydropyridines C-Glycosylated under mild condition. We prepared it by ultrasound irradiation method in an ionic liquid which leads to an efficient and eco-friendly method.

\section{Experimental}

\subsection{Materials}

All the reagents were purchased from Merck Chemical Company. IR spectra were recorded on a Perkin-Elmer 297 FT-IR spectroscopy. H-NMR, C-NMR and DEPT were recorded on Broker 300 and $500 \mathrm{MHz}$ spectroscopy. The MS spectra were obtained on a JEOL JMS HX-110 mass spectroscopy. Column chromatography was conducted with silica gel 230 - 400 mesh (Merck). Reactions were monitored by thin layer chromatography (TLC).

\subsection{Synthesis of Glycosylated Aldehyde}

\section{1-(6-Methoxy-2,2-dimethyl-tetrahydro-furo [3, 4-d] [1, 3] dioxol-4-yl)-} ethane-1,2-diol

A mixture of D-mannose (5 g, $27.77 \mathrm{mmol}), 2$,2-dimethylpropane (17 $\mathrm{ml})$, acetone $(16.5 \mathrm{ml})$, methanol $(16.5 \mathrm{ml})$ and concentrated $\mathrm{HCl}(0.5 \mathrm{ml})$ was refluxed for $2 \mathrm{~h}$. The water was added and the solution concentrated under reduced pressure. Methanol $(50 \mathrm{ml})$ and concentrated $\mathrm{HCl}(1.25 \mathrm{ml})$ was then added. The mixture was stirred at room temperature for $3 \mathrm{~h}$, neutralized with a saturated sodium hydrogen carbonate solution. The residue was taken in hot chloroform and the solution was filtered. The product was methyl-2,3-O-isopropylidenemannofuranoside as a oil colorless $(5.43 \mathrm{~g}, 83 \%)$.

H-NMR (300 MHz, $\left.\mathrm{CDCl}_{3}\right): \delta=4.87(\mathrm{~s}, 1 \mathrm{H}), 4.72(\mathrm{~d}, 1 \mathrm{H}), 4.49$ (d, 1H), 3.12 (brs, $1 \mathrm{H}), 4,30$ (dd, 1H), 3,57 (dd, 1H), 3.61 (dd, 1H), $3.32\left(\mathrm{~s}, \mathrm{OCH}_{3}, 3 \mathrm{H}\right), 1.37$ $\left(\mathrm{s}, \mathrm{CH}_{3}, 3 \mathrm{H}\right), 1.22\left(\mathrm{~s}, \mathrm{CH}_{3}, 3 \mathrm{H}\right)$ as shown in Figure A1. C-NMR (500 MHz, $\left.\mathrm{CDCl}_{3}\right): 113,10764.8,80.4,79.5,70.7,85.1,54.9,26.3,25.1$ as shown in Figure A2. FTIR: $3448,1638,1084 \mathrm{~cm}^{-1}$ as shown in Figure A3. 


\section{6-Methoxy-2,2-dimethyl-tetrahydro furo [3, 4-d] [1, 3] dioxole-4- carbaldehyde}

A solution of sodium periodate $(5.46 \mathrm{~g} 25.52 \mathrm{mmol})$ in water was added to methyl-2,3-O-isopropylidene-mannofuranoside $(5.43 \mathrm{~g})$ in methanol $(150 \mathrm{ml})$. In room temperature the mixture was stirred and dried under reduced pressure. The residue was filtered and evaporated to give the aldehyde (4.1 $\mathrm{g} 75 \%)$.

H-NMR (300 MHz, $\left.\mathrm{CDCl}_{3}\right): \delta=9.5(\mathrm{~s}, 1 \mathrm{H}), 5.1(\mathrm{~s}, 1 \mathrm{H}), 5.08(\mathrm{~d}, 1 \mathrm{H}), 4.63(\mathrm{~d}$, $1 \mathrm{H}), 4.38(\mathrm{~d}, 1 \mathrm{H}), 3.36\left(\mathrm{~s}, \mathrm{OCH}_{3}, 3 \mathrm{H}\right), 1.44\left(\mathrm{~s}, \mathrm{CH}_{3}, 3 \mathrm{H}\right), 1.30\left(\mathrm{~s}, \mathrm{CH}_{3}, 3 \mathrm{H}\right)$ as shown in Figure A4; C-NMR (500 MHz, $\mathrm{CDCl}_{3}$ ): 200.7, 109.1, 89.4, 84.4, 80.7, 55.6, 25.8, 24.8 as shown in Figure A5; FTIR: 3448, 1638, $1088 \mathrm{~cm}^{-1}$.

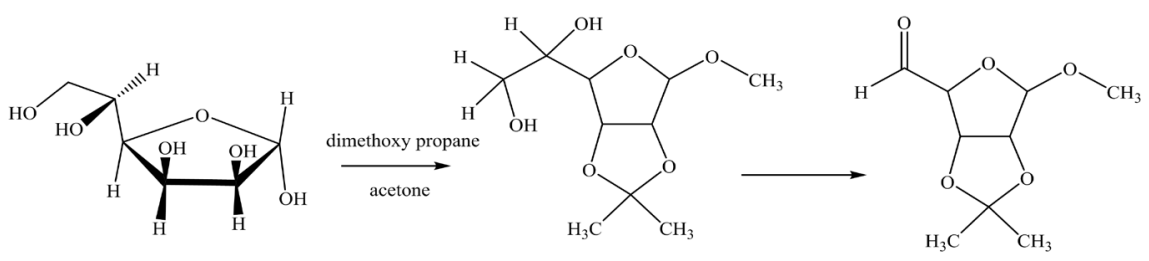

\subsection{Synthesis of 1,4-Dihydropyridines Derivative}

\section{Hantzsch reaction:}

A solution of glycosylated aldehyde compound (1.08 g, $2.5 \mathrm{mmol})$ and ethyl aceto acetate $(0.65 \mathrm{~g}, 2.5 \mathrm{mmol})$ in the presence of $0.1 \mathrm{ml}$ piperidine and $0.25 \mathrm{ml}$ glacial acetic acid in $50 \mathrm{ml}$ methanol was refluxed for $72 \mathrm{~h}$. after removing the solvent, the reaction mixture was refluxed with ethyl acetoacetate $(0.65 \mathrm{~g}, 2.5$ mmol) ammonium acetate $(1.2 \mathrm{~g})$ for $12 \mathrm{~h}$. The residue was purified and the product was crystallized to give $426 \mathrm{mg}$ (40\%) of 4-(3, 4-Dihydroxy-5-methoxytetrahydro-furan-2-yl)-2,6-dimethyl-1,4-dihydro-pyridine-3,5-dicarboxylic acid 3-ethyl ester 5-methyl ester.

H-NMR (300 MHz, $\mathrm{CDCl}_{3}$ ): $\delta=5.42(\mathrm{~s}, \mathrm{H}), 4.53(\mathrm{~s}, 1 \mathrm{H}), 4.19$ (d, $\left.\mathrm{CH} 2\right)$, 4.13(d, $1 \mathrm{H}), 3.24\left(\mathrm{~d}, \mathrm{CH}_{3}\right), 1.71\left(\mathrm{~s}, \mathrm{CH}_{3}, 3 \mathrm{H}\right), 1.41\left(\mathrm{~s}, \mathrm{CH}_{3}, 3 \mathrm{H}\right), 1.30\left(\mathrm{~s}, \mathrm{CH}_{3}, 3 \mathrm{H}\right)$ as shown in Figure A6; C-NMR $\left(500 \mathrm{MHz}, \mathrm{CDCl}_{3}\right)$ : $165,141.2,108.5,102.4$, $101.9,79.9,73.3,66.4,59.9,48.3,28.6,24.0$ as shown in Figure A7, MW=425, $\mathrm{C}_{21} \mathrm{H}_{31} \mathrm{NO}_{8}$ as shown in Figure A9.

\subsection{Ultrasound Accelerated Synthesis}

A mixture of ethyl acetoacetate $(0.259 \mathrm{~g}, 2 \mathrm{mmol})$, glycosylated aldehyde $(0.5 \mathrm{~g}$ $1.25 \mathrm{mmol})$, ammonium acetate $(0.154 \mathrm{~g}, 2 \mathrm{mmol})$ and ionic liquid [bmim] $\mathrm{BF}_{4}$ $(0.120 \mathrm{~g} 0.5 \mathrm{mmol})$ was successively charged into a screw-capped vial. The mixture was irradiated in a water bath for two hours. The reaction mixture was washed with water. The crude product purified by silica gel column chromatography using dichloromethane: ethyl acetate (98:2). The crystallized product yield was $50 \%$.

\section{Results and Discussion}

The original Hantzsch synthesis is usually completed via one-pot thermal cyclo- 
condensation of the reaction of a $\beta$-diketone with an aldehyde and ammonia in acetic acid which leads to dihyropyridine derivatives (Scheme 1). Compounds have different pharmacophores groups to prepare by Hantzsch cyclocondensation.

A Dihydropyridines C-Glycosylated has been prepared by a three-component reaction of $\beta$-keto ester, examines and glycosylated aldehyde (Scheme 2). Glycosylated aldehyde could undergo the Hantzsch cyclo condensation reaction effectively to afford the corresponding products in good yields.

The compounds were obtained as a solid, and its molecular formula, $\mathrm{C}_{21} \mathrm{H}_{31} \mathrm{NO}_{8}$, was established by MS $(\mathrm{m} / z$ 252,196). The proposed mechanism of Dihydropyridines C-Glycosylated synthesis showed in Scheme 3. The structure of compound was assigned by NMR spectroscopy (Table 1).<smiles>[R]OC(=O)CC(=O)[18OH]</smiles><smiles>C=O</smiles><smiles>[R]C1=C([R])C([R])C([R])=C(N)N1</smiles>

Scheme 1. Hantzsch cyclocondensation reaction.<smiles>CCCC(=O)C1=C(C)NC(C)=C(C(=O)CC)C1C1C2OC(C)(C)OC2C(OC)OC1C1OC(OC)C(O)C1O</smiles>

Scheme 2. Synthesis of C-Glycosylated 1, 4-Dihydropyridine.

Table 1. 1H NMR and 13C NMR Data for compound (CDCl3, $500 \mathrm{MHz}, \mathrm{ppm}, \mathrm{J} / \mathrm{Hz})$.

\begin{tabular}{|c|c|c|c|c|c|}
\hline $\mathrm{C}$ atom & $\square \delta_{\mathrm{H}}$ & $\delta_{\mathrm{C}}$ & $\mathrm{C}$ atom & $\delta_{\mathrm{H}}$ & $\delta_{\mathrm{C}}$ \\
\hline $\mathrm{C} 2-\mathrm{CH} 3$ & $2.25 \mathrm{~s}(3 \mathrm{H})$ & 19.1 & $\mathrm{C} 2$ & - & 144.1 \\
\hline $\mathrm{C} 6-\mathrm{CH} 3$ & $2.32 \mathrm{~s}(3 \mathrm{H})$ & 18.5 & $\mathrm{C} 6$ & - & 144.3 \\
\hline $\mathrm{H}-4^{\prime}$ & $\begin{array}{c}3.60 \mathrm{dd}(1 \mathrm{H}) \\
(\mathrm{J}=8.15-2.45)\end{array}$ & 79.7 & $\mathrm{C} 2$ & - & 112.4 \\
\hline H-6' & $4.7 \mathrm{~s}(1 \mathrm{H})$ & 106.6 & $\mathrm{C} 3$ & - & 100.9 \\
\hline $\mathrm{H}-7^{\prime}$ & $\begin{array}{c}4.41 \mathrm{~d}(1 \mathrm{H}) \\
(\mathrm{J}=5.8)\end{array}$ & 85.3 & C5 & - & 99.98 \\
\hline H-8' & $\begin{array}{l}4.49 \mathrm{dd}(1 \mathrm{H}) \\
(\mathrm{J}=5.8,2.7)\end{array}$ & 81.8 & $\mathrm{CO}$ & - & 168.1 \\
\hline $\mathrm{C} 2$ '- $\mathrm{CH} 3$ & $1.259 \mathrm{~s}(3 \mathrm{H})$ & 26.3 & $\mathrm{CO}$ & - & 168.4 \\
\hline $\mathrm{C} 2$ '- $\mathrm{CH} 3$ & $1.440 \mathrm{~s}(3 \mathrm{H})$ & 25.8 & CH3(side group) & $1.309 \mathrm{t}(3 \mathrm{H})$ & 14.29 \\
\hline C6'-OCH3 & $3.17 \mathrm{~s}(3 \mathrm{H})$ & 53.9 & $\mathrm{CH} 3$ (side group) & $1.284 \mathrm{t}(3 \mathrm{H})$ & 14.22 \\
\hline \multirow[t]{2}{*}{$\mathrm{H}-4$} & $\begin{array}{l}4.64 \mathrm{~d}(1 \mathrm{H}) \\
(\mathrm{J}=8.15)\end{array}$ & 33.6 & $\mathrm{CH} 2$ (side group) & $4.1 \mathrm{~m}(2 \mathrm{H})$ & 59.7 \\
\hline & & & CH2 (side group) & $4.1 \mathrm{~m} \mathrm{(2H)}$ & 59.4 \\
\hline
\end{tabular}



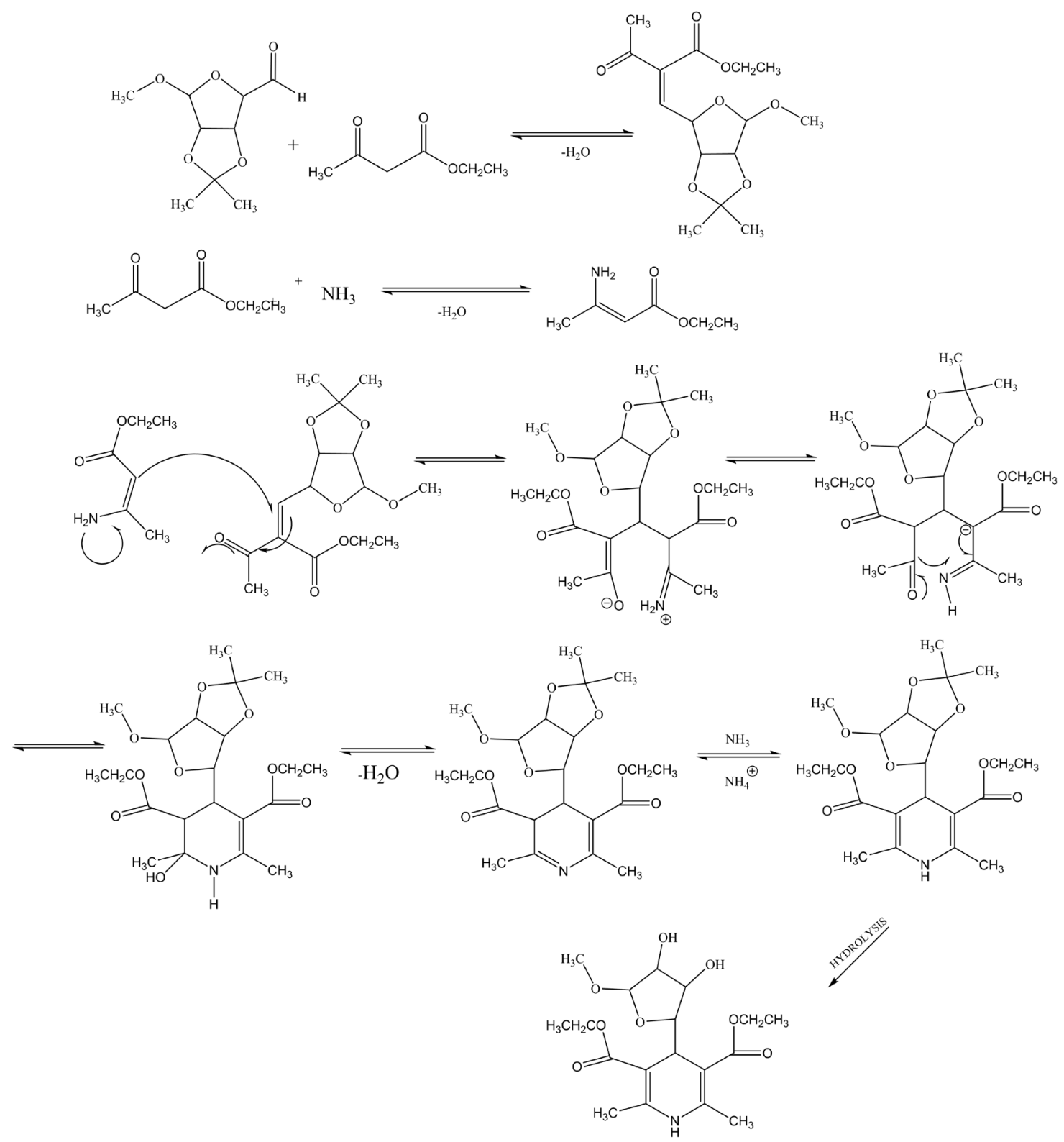

Scheme 3. Proposed mechanism of Dihydropyridines C-Glycosylated.

The H-NMR spectrum showed characteristic resonances for two methyl groups as singlets at 2.25 and 2.32 , one allylic resonance at $4.64(1 \mathrm{H}, \mathrm{q}, \mathrm{J}=7.2$ $\mathrm{Hz}$ ), and four $\mathrm{CH}$ resonances at $3.60(1 \mathrm{H}, \mathrm{dd}, \mathrm{J}=8.15,2.45 \mathrm{~Hz}), 4.7(1 \mathrm{H}, \mathrm{s}), 4.41$ $(1 \mathrm{H}, \mathrm{d}, \mathrm{J}=5.8 \mathrm{~Hz})$ and $4.49(1 \mathrm{H}, \mathrm{dd}, \mathrm{J}=5.8,2.7 \mathrm{~Hz})$.

The C-NMR resonances showed 21 carbon signals, which could be resolved by DEPT experiments into seven $\mathrm{CH} 3$ groups at 19.1 (C-2), 18.5 (C-6), 26.3 (C-2'), $25.8(\mathrm{C}-2)$ ') $53.9\left(\mathrm{C}-\mathrm{OCH}_{3}\right) 14.29$ and $14.22\left(\mathrm{CH}_{3}\right.$ side group), two $\mathrm{CH}_{2}$ groups at 59.7 and $59.4\left(\mathrm{CH}_{2}\right.$ side group $)$, five $\mathrm{CH}$ groups at $33.6(\mathrm{C}-4), 79.9\left(\mathrm{C}-4^{\prime}\right), 81.8$ (C-8'), 85.3 (C-7') 106.6 (C-6'), and seven quaternary carbons at 99.8 (C-5), 100.9 (C-3 ), 112.4 (C-2), 144.1 (C-2), 144.3 (C-6),168.1 (CO),168.4 (CO) as two carbonyl functional groups as shown in Figure A8. 
<smiles></smiles>

Using ultrasound method with an ionic liquid [brim] $\mathrm{BF}_{4}$ or 1-butyl-3-methyl imidazolium tetrafluoroborate as promoter for the synthesis of Hantzsch 1,4-dihydropyridines does not present improvement at room temperature, but the reaction times are considerably shorter $(2.30 \mathrm{~h})$ compared to classical synthesis. After the reaction was complete, the reaction mixture is simply washed with water and the ionic liquid is isolated from the product. The ionic liquid is recyclable as reaction medium.

\section{Conclusion}

We present a novel Dihydropyridines C-Glycosylated by Hantzsch cyclocondensation in which the glycosylated aldehyde was designed. We carried out an ultrasound-accelerated synthesis of compound in an ionic liquid. The significant improvements offered by this procedure are: fast reaction, simple operation and mild reaction conditions (room temperature) and green aspects avoiding organic solvents and toxic catalyst.

\section{Acknowledgements}

This research was supported by research department of Islamic Azad University-north Tehran branch.

\section{References}

[1] Sridharan, V., Perumal, P.T., Avendano, C. and Menendez, J.C. (2007) A New Three-Component Domino Synthesis of 1,4-Dihydropyridines. Tetrahedron, 63, 4407-4413. https://doi.org/10.1016/j.tet.2007.03.092

[2] Kumar, A. and Maurya, R.A. (2003) Organocatalysed Three-Component Domino Synthesis of 1,4-Dihydropyridines under Solvent Free Conditions. Tetrahedron, 64, 3477-3482. https://doi.org/10.1016/j.tet.2008.02.022

[3] Tewari, N., Dwivedi, N. and Tripathi, R.P. (2004) Tetrabutylammonium Hydrogen Sulfate Catalyzed Eco-Friendly and Efficient Synthesis of Glycosyl 1,4-Dihydropyridines. Tetrahedron Letters, 45, 9011-9014. https://doi.org/10.1016/j.tetlet.2004.10.057

[4] Sabitha, G., Kumar Reddy, G.S.K., Reddy, Ch.S. and Yadav, J.S. (2003) A Novel TMSI-Mediated Synthesis of Hantzsch 1,4-Dihydropyridines at Ambient Temperature. Tetrahedron Letters, 44, 4129-4131. https://doi.org/10.1016/S0040-4039(03)00813-X

[5] Moreau, J., Duboc, A., Hubert, C., Hurvois, J.P. and Renaud, J.L. (2007) Metal-Free Brønsted Acids Catalyzed Synthesis of Functional 1,4-Dihydropyridines. Tetrahedron Letters, 48, 8647-8650. https://doi.org/10.1016/j.tetlet.2007.10.040 
[6] Barton, D.H.R., Gero, S.D., Quiclet-Sire, B. and Samadi, M. (1994) Stereocontrolled Radical Reactions in Carbohydrate and Nucleoside Chemistry. Tetrahedron Asymmetry, 5, 2123-2136. https://doi.org/10.1016/S0957-4166(00)86288-6

[7] Dondoni, A., Massi, A. and Minghini, E. (2002) Dihydropyridine $C$-Glycoconjugates by Hantzsch Cyclocondensation. Synthesis of a C(6)-Glycosylated Nifedipine Analogue. Helvetica Chimia Acta, 85, 3331-3348. https://doi.org/10.1002/1522-2675(200210)85:10<3331::AID-HLCA3331>3.0.CO;2$\underline{\mathrm{X}}$

[8] Dondoni, A. and Massi, A. (2003) Decoration of Dihydropyrimidine and Dihydropyridine Scaffolds with Sugars via Biginelli and Hantzsch Multicomponent Reactions: An Efficient Entry to a Collection of Artificial Nucleosides. Molecular Diversity, 6, 261-270. https://doi.org/10.1023/B:MODI.0000006806.91483.a3

[9] Dondoni, A., Massi, A., Sabbatini, S. and Bertolasi, V. (2002) Three-Component Biginelli Cyclocondensation Reaction Using $C$-Glycosylated Substrates. Preparation of a Collection of Dihydropyrimidinone Glycoconjugates and the Synthesis of C-Glycosylated Monastrol Analogues. Journal of Organic Chemistry, 67, 6979-6994. https://doi.org/10.1021/jo0202076

[10] Datar, P.A. and Auti, P.B. (2016) Design and Synthesis of Novel 4-Substituted 1,4-Dihydropyridine Derivatives as Hypotensive Agents. Journal of Saudi Chemical Society, 20, 510-516. https://doi.org/10.1016/j.jscs.2012.08.003

[11] Wang, S.X., Li, Z.Y., Zhang, J.C. and Li, J.T. (2008) The Solvent-Free Synthesis of 1,4-Dihydropyridines under Ultrasound Irradiation without Catalyst. Ultrasonic Sonochemistry, 15, 677-680. https://doi.org/10.1016/j.ultsonch.2008.02.009

[12] Sridhar, R. and Perumal, P.T. (2005) A New Protocol to Synthesize 1,4-Dihydropyridines by Using 3,4,5-Trifluorobenzeneboronic Acid as a Catalyst in Ionic Liquid: Synthesis of Novel 4-(3-Carboxyl-1 $H$-pyrazol-4-yl)-1,4-dihydropyridines $T e$ trahedron, 61, 2465-2470.

[13] Legeay, J.C., Eynde, J.J.V. and Bazureau, J.P. (2005) Ionic Liquid Phase Technology Supported the Three Component Synthesis of Hantzsch 1,4-Dihydropyridines and Biginelli 3,4-Dihydropyrimidin-2(1H)-ones under Microwave Dielectric Heating. Tetrahedron, 61, 12386-12397. https://doi.org/10.1016/j.tet.2005.09.118

[14] Kumar, J.A., Shridhar, G., Ladage, S. and Ravishankard, L. (2016) Synthesis of 1,4-Dihydropyridine Esters Using Low-Melting Sugar Mixtures as Green Solvents. Synthethic Commnucation, 9, 438. 


\section{Supporting Information}

\section{Spectral and analytical data of compounds:}

1-(6-Methoxy-2,2-dimethyl-tetrahydro-furo [3,4- $d][1,3]$ dioxol-4-yI)-ethane-1, 2-diol (1)

Yield: $5.60 \mathrm{~g}$ (85\%). IR: $3448,1638,1084 \mathrm{~cm}^{-1} .{ }^{1} \mathrm{H}-\mathrm{NMR}\left(300 \mathrm{MHz}, \mathrm{CDCl}_{3}\right): \delta$ $=4.87(\mathrm{~s}, 1 \mathrm{H}), 4.72(\mathrm{~d}, 1 \mathrm{H}), 4.49(\mathrm{~d}, 1 \mathrm{H}), 3.12(\mathrm{brs}, 1 \mathrm{H}), 4,30(\mathrm{dd}, 1 \mathrm{H}), 3,57(\mathrm{dd}$, $1 \mathrm{H}), 3.61(\mathrm{dd}, 1 \mathrm{H}), 3.32\left(\mathrm{~s}, \mathrm{OCH}_{3}, 3 \mathrm{H}\right), 1.37\left(\mathrm{~s}, \mathrm{CH}_{3}, 3 \mathrm{H}\right), 1.22\left(\mathrm{~s}, \mathrm{CH}_{3}, 3 \mathrm{H}\right)$. ${ }^{13} \mathrm{C}-\mathrm{NMR}\left(500 \mathrm{MHz}, \mathrm{CDCl}_{3}\right.$ ): 113, 107 64.8, 80.4, 79.5, 70.7, 85.1, 54.9, 26.3, 25.1.

6-Methoxy-2,2-dimethyl-tetrahydro-furo $[3,4-d][1,3]$ dioxole-4-carbaldehyde (2)

Yield: $4.0 \mathrm{~g}(75 \%)$.IR: 3448, 1638, $1088 \mathrm{~cm}^{-1} .{ }^{1} \mathrm{H}-\mathrm{NMR}\left(300 \mathrm{MHz}, \mathrm{CDCl}_{3}\right): \delta=$ $9.5(\mathrm{~s}, 1 \mathrm{H}), 5.1(\mathrm{~s}, 1 \mathrm{H}), 5.08(\mathrm{~d}, 1 \mathrm{H}), 4.63(\mathrm{~d}, 1 \mathrm{H}), 4.38(\mathrm{~d}, 1 \mathrm{H}), 3.36\left(\mathrm{~s}, \mathrm{OCH}_{3}, 3 \mathrm{H}\right)$, 1.44(s, $\left.\mathrm{CH}_{3}, 3 \mathrm{H}\right), 1.30\left(\mathrm{~s}, \mathrm{CH}_{3}, 3 \mathrm{H}\right){ }^{13} \mathrm{C}-\mathrm{NMR}\left(500 \mathrm{MHz}, \mathrm{CDCl}_{3}\right): 200.7,109.1$, $89.4,84.4,80.7,55.6,25.8,24.8$

4-(3,4-Dihydroxy-5-methoxy-tetrahydro-furan-2-yI)-2,6-dimethyl-1,4-dihydr o-pyridine-3,5-dicarboxylic acid 3-ethyl ester 5 -methyl ester. (3)

Yield: (40\%),mp.185-190 C ${ }^{1} \mathrm{H}-\mathrm{NMR}(300 \mathrm{MHz}, \mathrm{CDCl} 3): \delta=5.42(\mathrm{~s}, \mathrm{H}), 4.53$ (s, $1 \mathrm{H}), 4.19\left(\mathrm{~d}, \mathrm{CH}_{2}\right), 4.13(\mathrm{~d}, 1 \mathrm{H}), 3.24\left(\mathrm{~d}, \mathrm{CH}_{3}\right), 1.71\left(\mathrm{~s}, \mathrm{CH}_{3}, 3 \mathrm{H}\right), 1.41\left(\mathrm{~s}, \mathrm{CH}_{3}\right.$, $3 \mathrm{H}), 1.30\left(\mathrm{~s}, \mathrm{CH}_{3}, 3 \mathrm{H}\right){ }^{13} \mathrm{C}-\mathrm{NMR}(500 \mathrm{MHz}, \mathrm{CDCl} 3): 165,141.2,108.5,102.4$, 101.9, 79.9, 73.3, 66.4, 59.9, 48.3, 28.6, 24.0., $\mathrm{MW}=425, \mathrm{C}_{21} \mathrm{H}_{31} \mathrm{NO}_{8}$. 


\section{Appendix}

11 HNMA in $\operatorname{CDC} 13$ at $298 \mathrm{~K} 86 / 7 / 25$
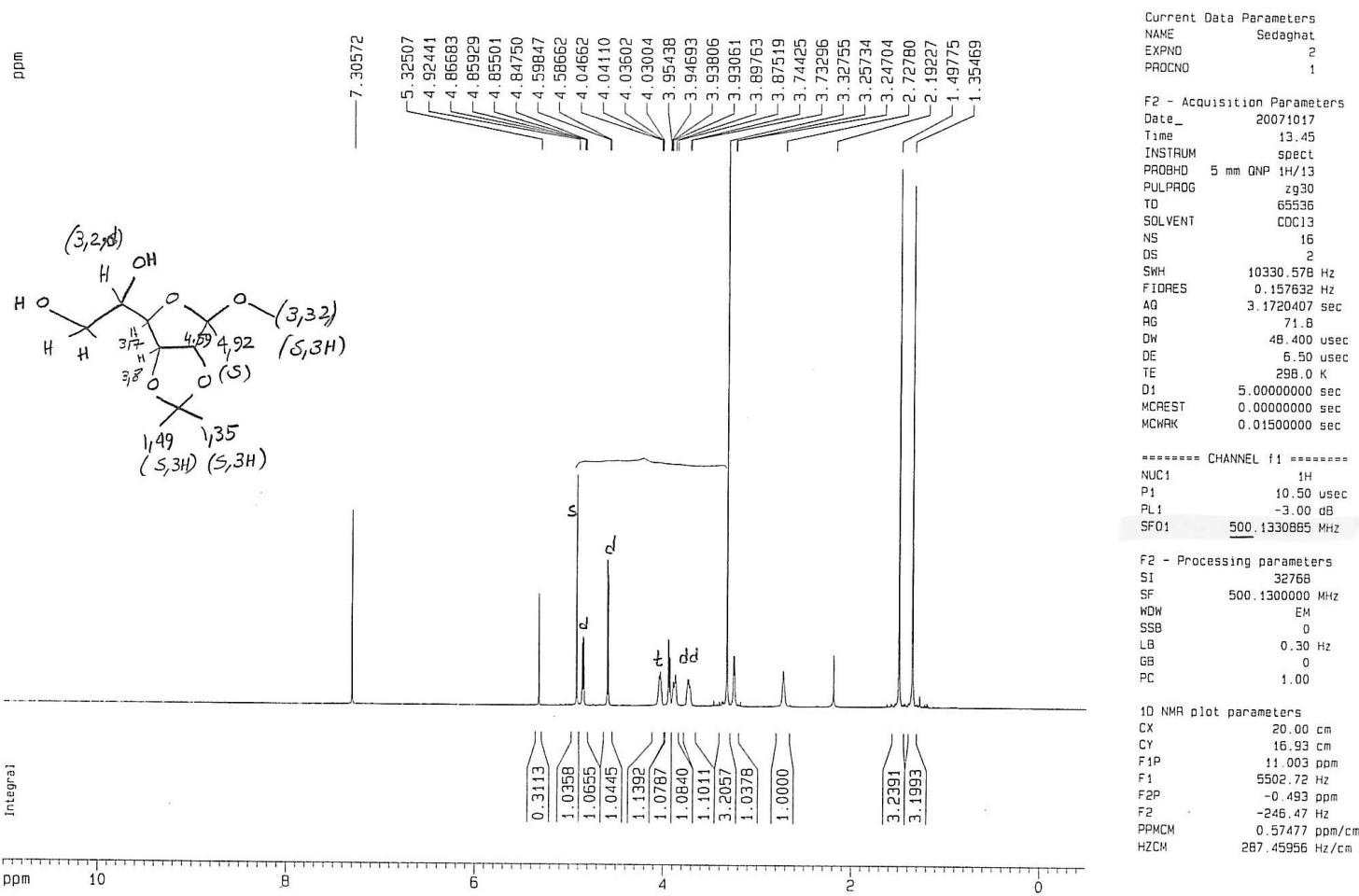

Figure A1. HNMR "methyl-2,3-O-isopropylidene-mannofuranoside".

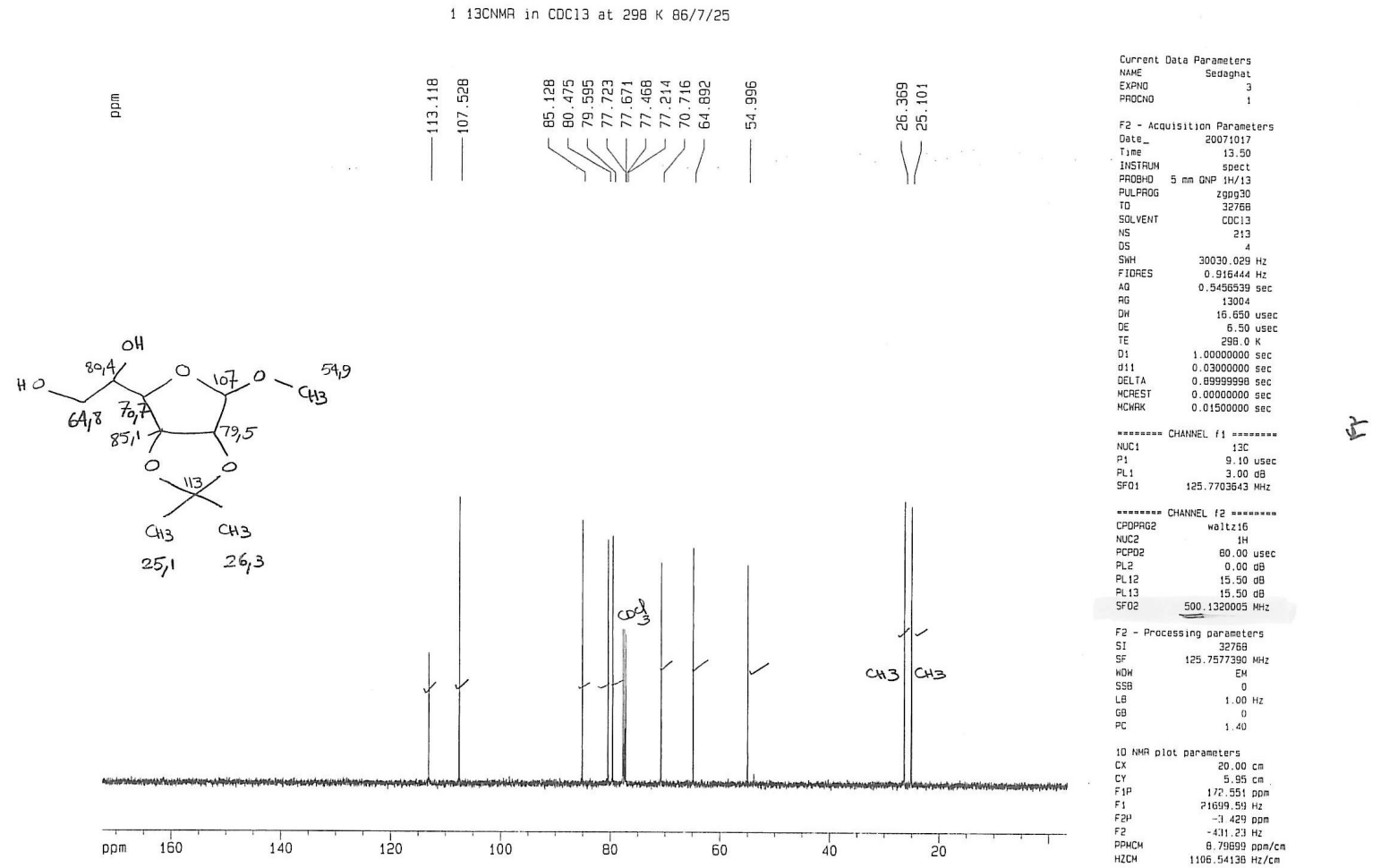

Figure A2. CNMR “methyl-2,3-O-isopropylidene-mannofuranoside”. 


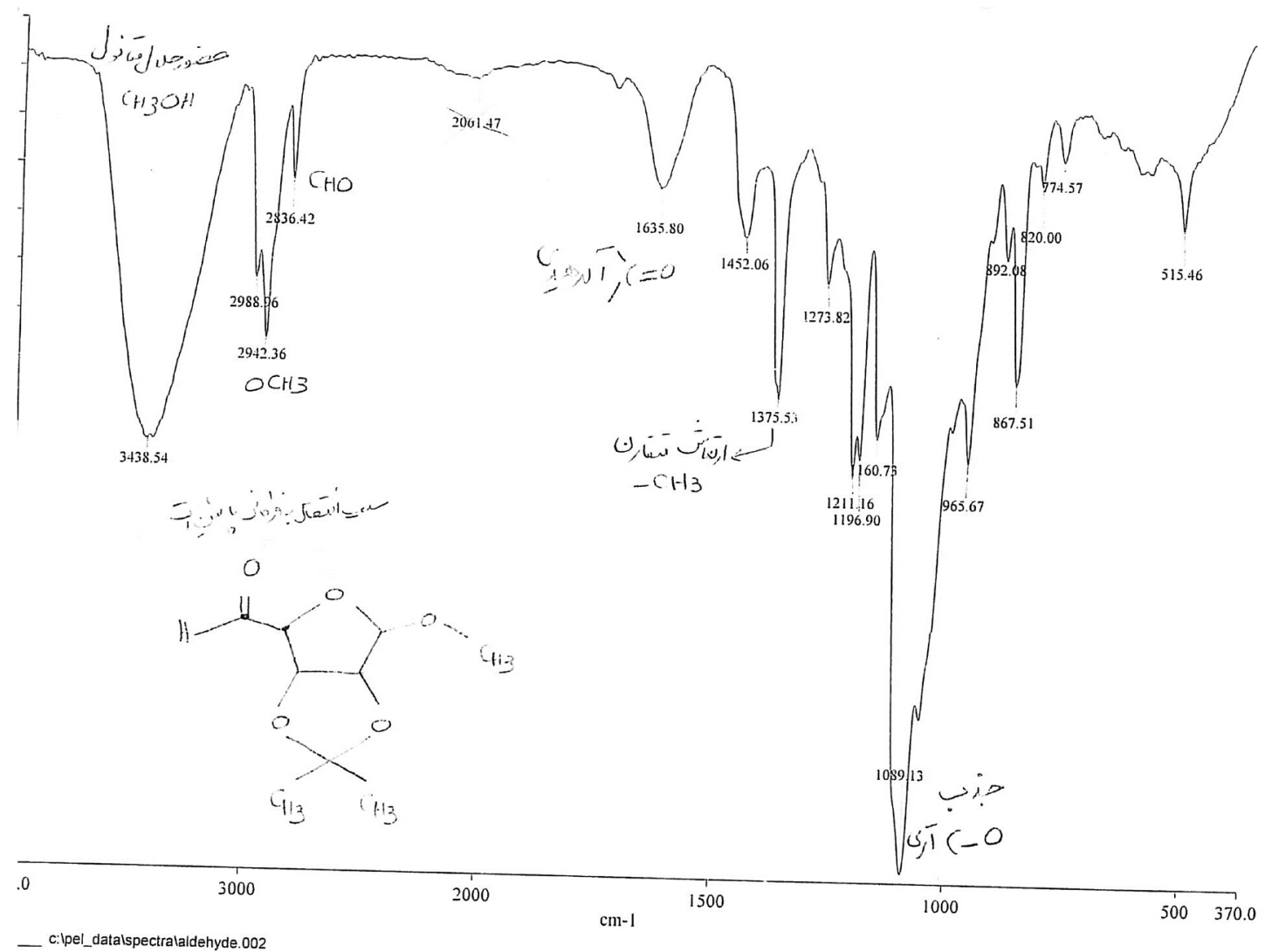

Figure A3. FTIR “6-Methoxy-2, 2-dimethyl-tetrahydro-furo [3, 4-d] [1, 3] dioxole-4-carbaldehyde".

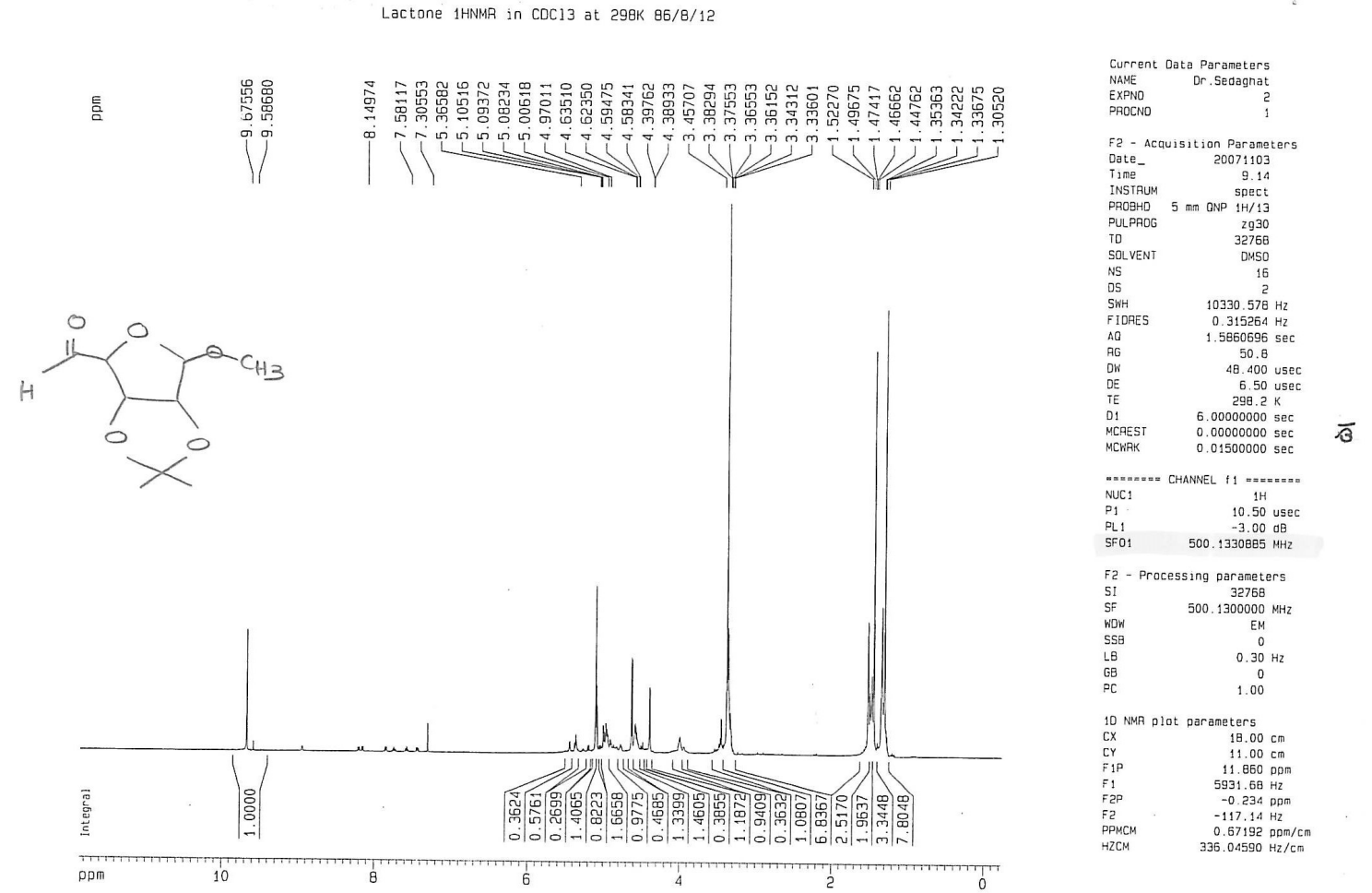

Figure A4. HNMR "6-Methoxy-2, 2-dimethyl-tetrahydro-furo [3, 4-d] [1, 3] dioxole-4-carbaldehyde". 


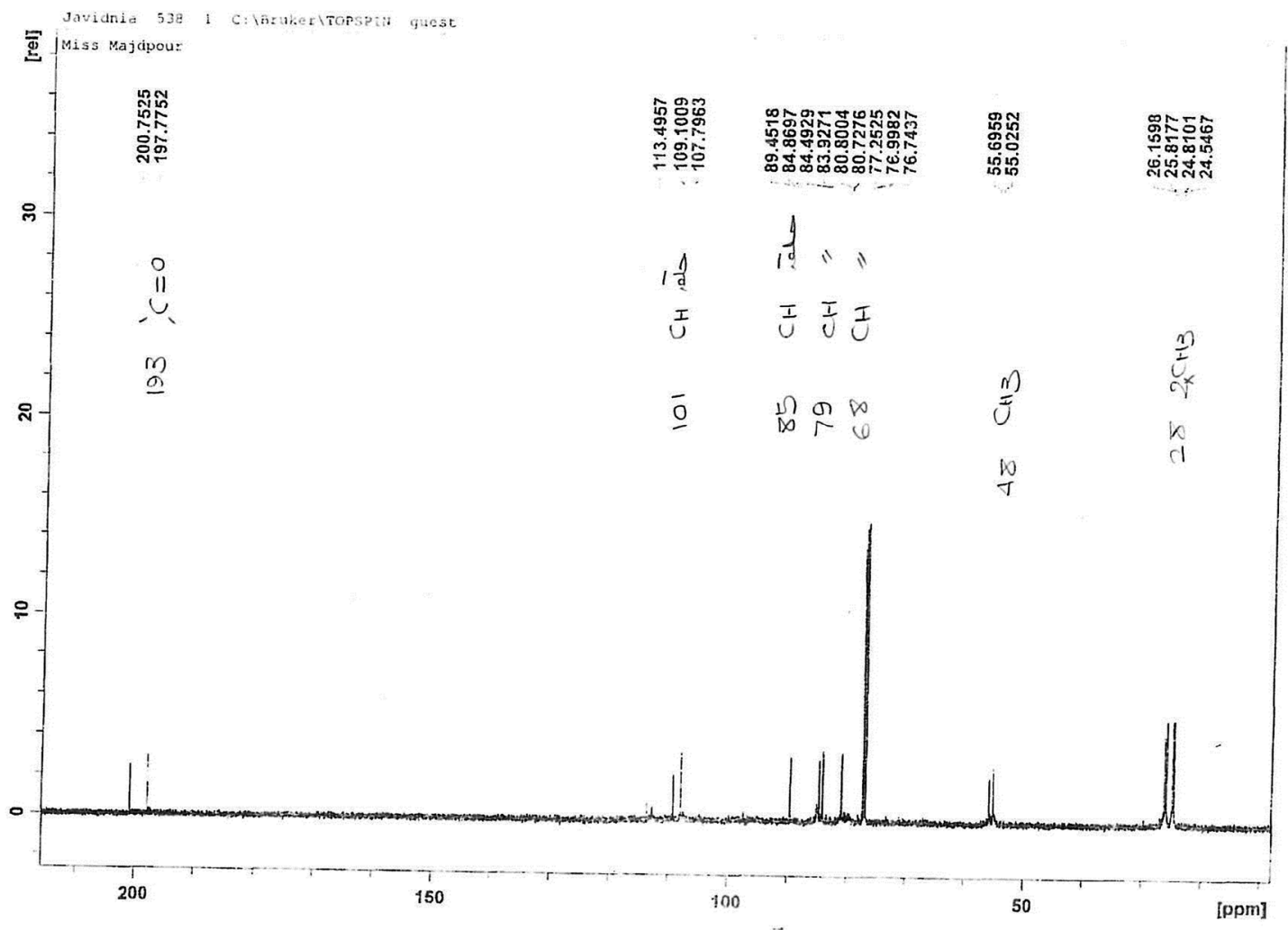

Figure A5. CNMR" 6-Methoxy-2, 2-dimethyl-tetrahydro-furo [3, 4-d] [1, 3] dioxole-4-carbaldehyde”.

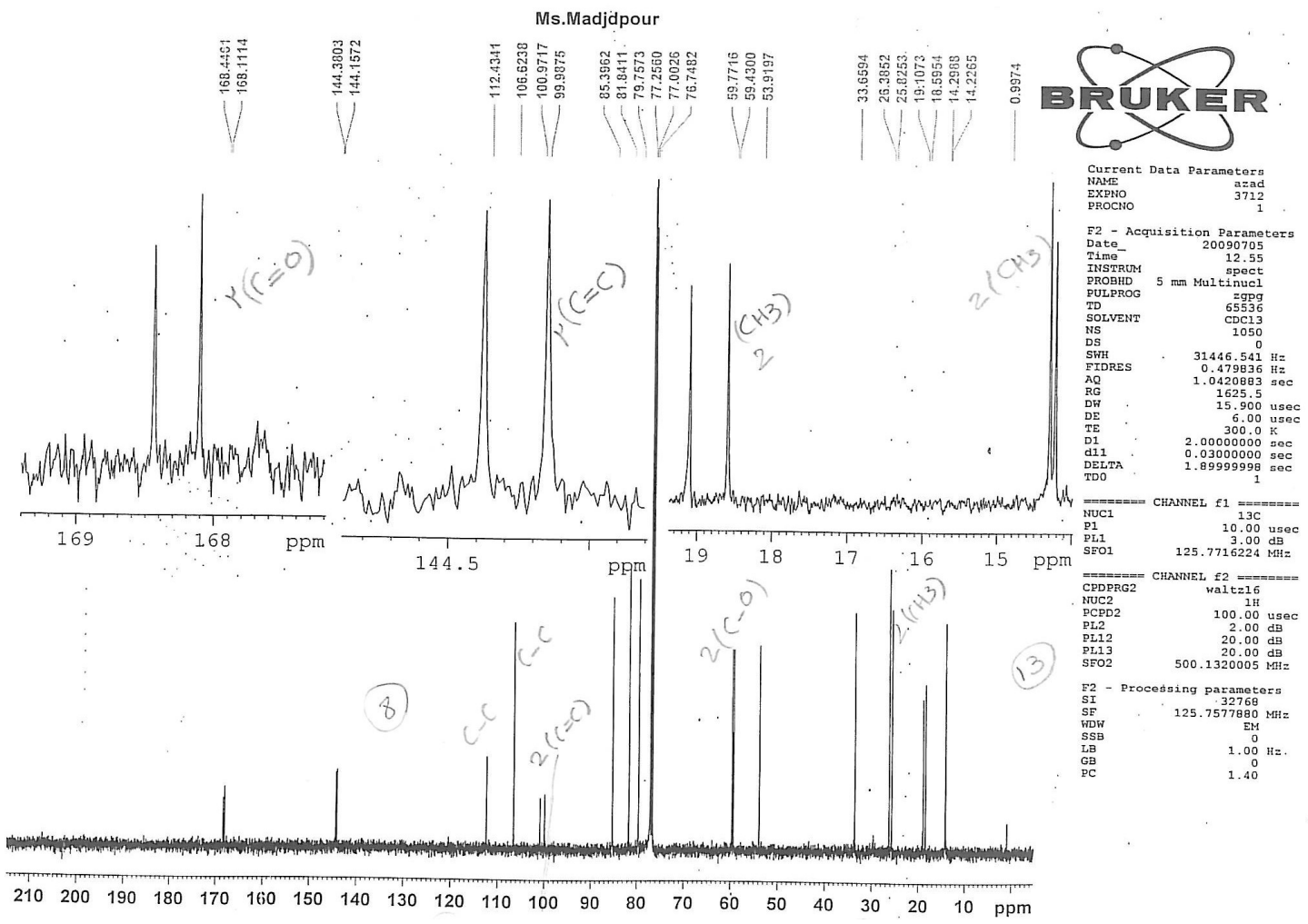

Figure A6. HNMR “4-(3,4-Dihydroxy-5-methoxy-tetrahydro-furan-2-yl)-2,6-dimethyl-1,4-dihydro-pyridine-3,5-dicarboxylic acid 3-ethyl ester 5-methyl ester”. 


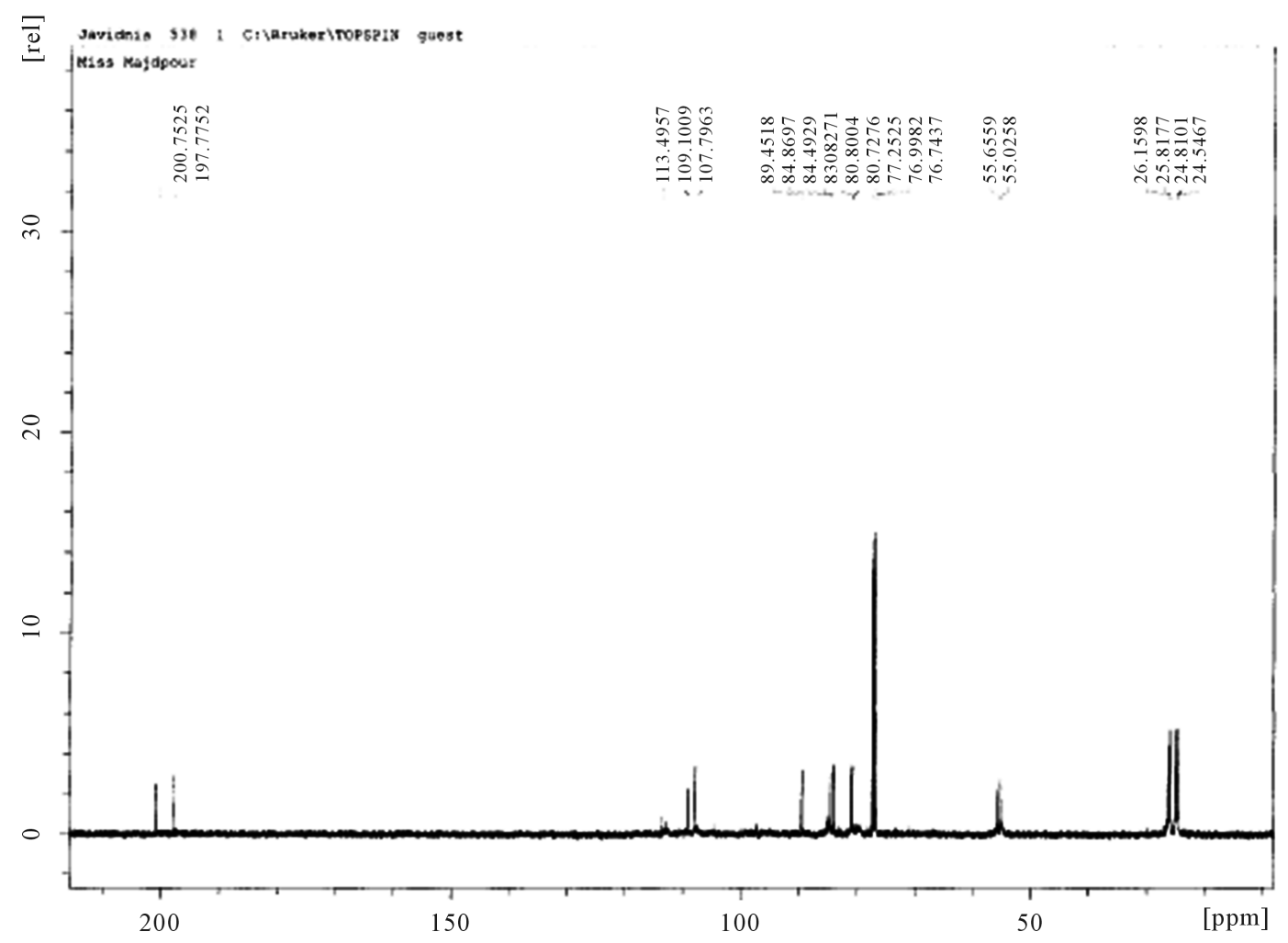

Figure A7. CNMR “4-(3, 4-Dihydroxy-5-methoxy-tetrahydro-furan-2-yl)-2,6-dimethyl-1,4-dihydro-pyridine-3,5-dicarboxylic acid 3-ethyl ester 5-methyl ester".
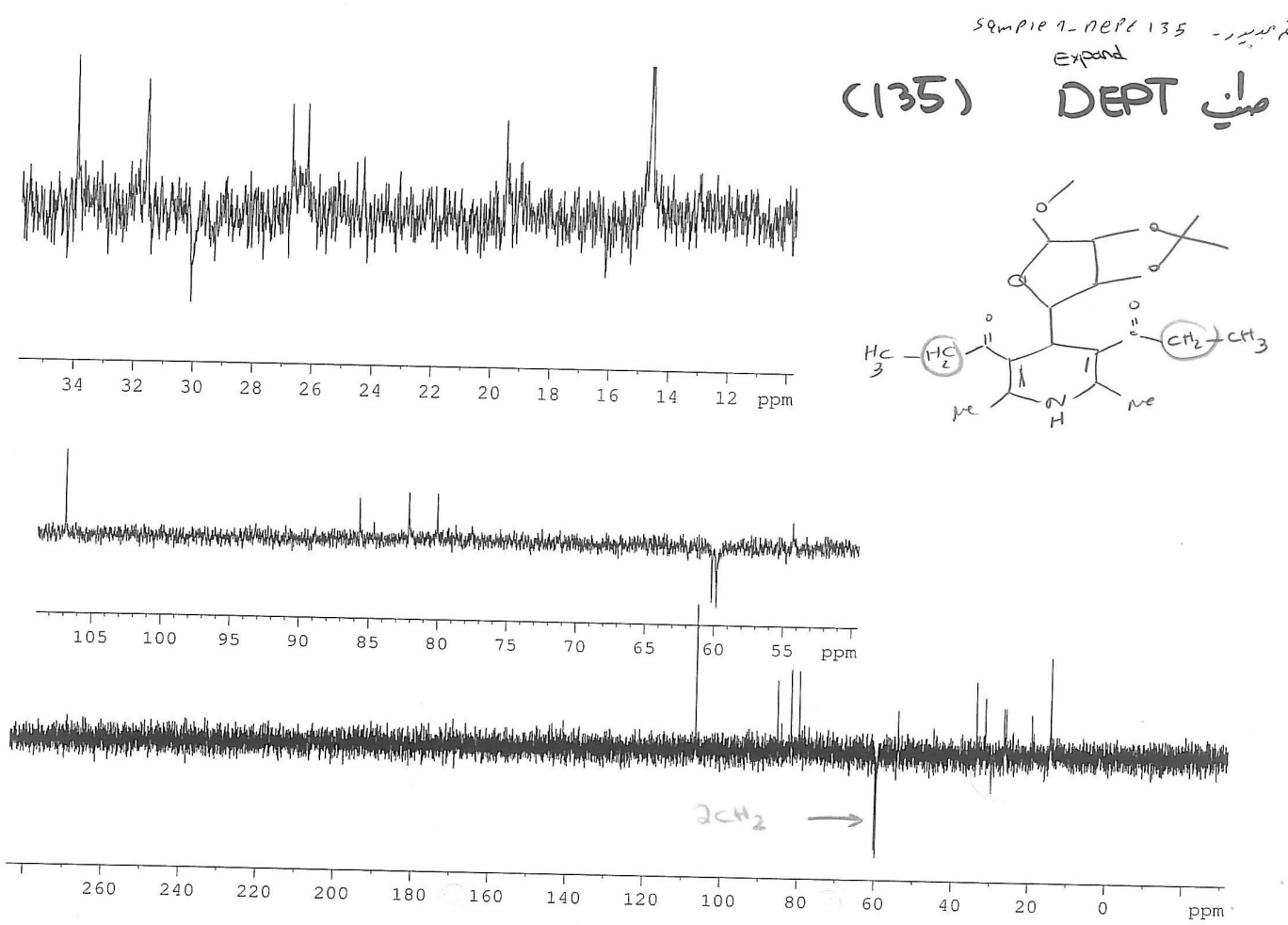

Figure A8. NMR-DEPT “4-(3,4-Dihydroxy-5-methoxy-tetrahydro-furan-2-yl)-2,6-dimethyl-1,4-dihydro-pyridine-3,5-dicarboxylic acid 3-ethyl ester 5-methyl ester". 
MAJDPUR 102 (1.555)

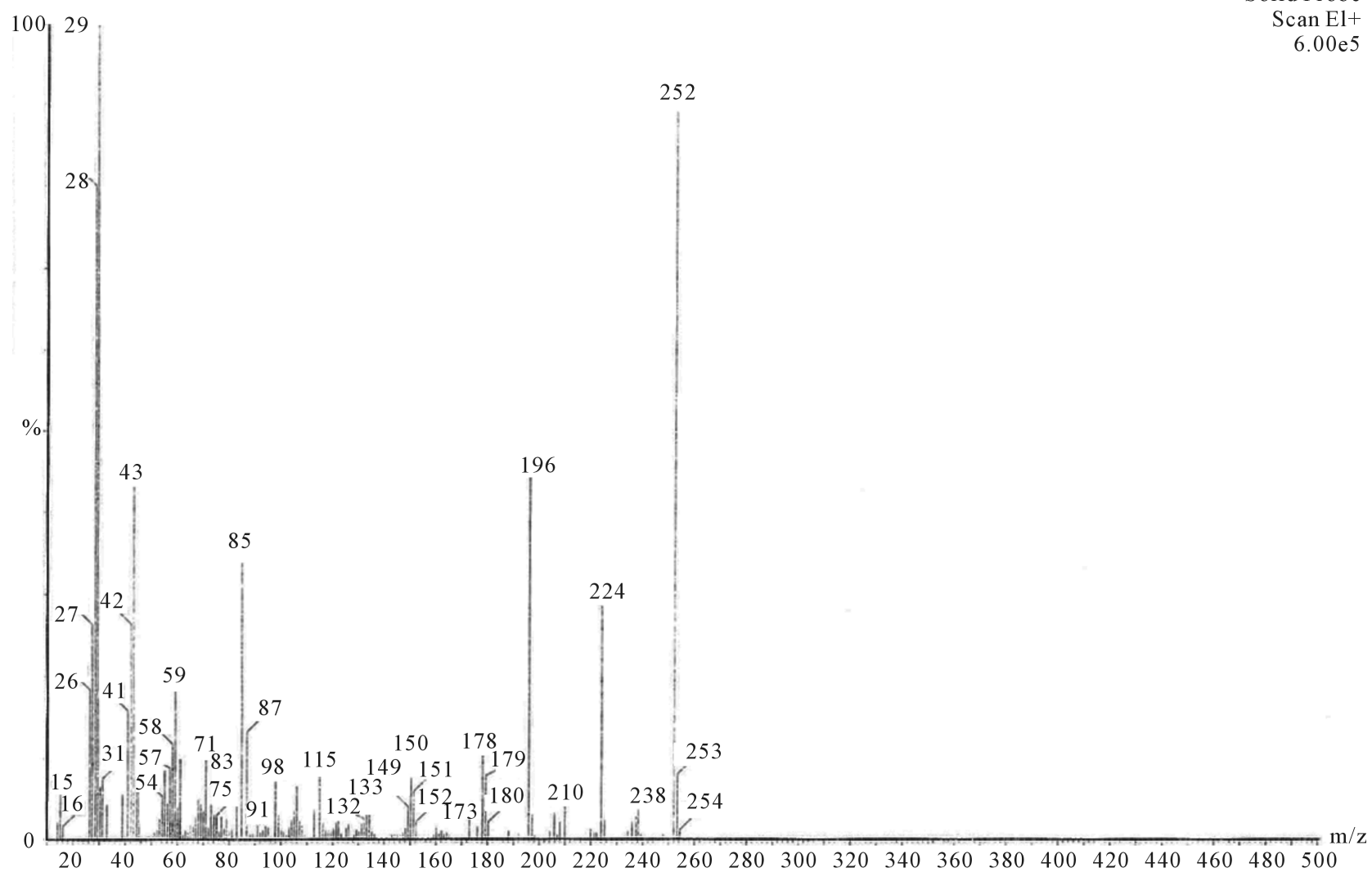

Figure A9. MS “4-(3,4-Dihydroxy-5-methoxy-tetrahydro-furan-2-yl)-2,6-dimethyl-1,4-dihydro-pyridine-3,5-dicarboxylic acid 3-ethyl ester 5-methyl ester". 